PROCEEDINGS OF THE

AMERICAN MATHEMATICAL SOCIETY

Volume 135, Number 6, June 2007, Pages 1775-1784

S 0002-9939(06)08659-X

Article electronically published on November 7, 2006

\title{
HOLES IN THE SPECTRUM OF FUNCTIONS GENERATING AFFINE SYSTEMS
}

\author{
JEAN-PIERRE GABARDO AND YUN-ZHANG LI
}

(Communicated by Joseph A. Ball)

\begin{abstract}
Given a $d \times d$ expansive dilation matrix $D$, a measurable set $E \subset$ $\mathbb{R}^{d}$ is called a $D^{t}$-dilation generator of $\mathbb{R}^{d}$ if $\mathbb{R}^{d}$ is tiled (modulo null sets) by the collection $\left\{\left(D^{t}\right)^{j} E, j \in \mathbb{Z}\right\}$. Our main goal in this paper is to prove certain results relating the support of the Fourier transform of functions generating a wavelet or orthonormal affine system associated with the dilation $D$ to an arbitrary set $E$ which is a $D^{t}$-dilation generator of $\mathbb{R}^{d}$.
\end{abstract}

\section{INTRODUCTION}

Let $d$ be a fixed positive integer. We denote by $\mathbb{Z}^{d}$ the set of points with integral coordinates in $\mathbb{R}^{d}$ and by $I_{d}$ the $d \times d$ identity matrix. The Fourier transform $\hat{f}$ of a function $f \in L^{1}\left(\mathbb{R}^{d}\right)$ is defined by

$$
\hat{f}(\xi)=\int_{\mathbb{R}^{d}} f(x) e^{-2 \pi i\langle\xi, x\rangle} d x, \quad \xi \in \mathbb{R}^{d},
$$

where $\langle.,$.$\rangle denotes the usual dot product in \mathbb{R}^{d}$. If $E$ is a measurable subset of $\mathbb{R}^{d}$, we will denote by $|E|$ its Lebesgue measure. A $d \times d$ matrix $D$ with real entries is called a dilation matrix if the moduli of all its eigenvalues are greater than 1 . Throughout this paper, $D^{t}$ denotes the transpose of $D$. If $B$ is a $d \times d$ matrix with real entries and $E$ is a subset of $\mathbb{R}^{d}$, we denote by $B E$ the set

$$
B E=\{B x, x \in E\} .
$$

If $B=\lambda I_{d}$, we use the notation $\lambda E$ instead. We point out that, unless otherwise specified, relations between two measurable sets in $\mathbb{R}^{d}$, such as equality or inclusion, are always understood up to a subset of zero Lebesgue measure. Similarly, equality or inequality between functions is always understood in the "pointwise almosteverywhere" sense. Given a $d \times d$ dilation matrix $D$ and any function $f$ defined on $\mathbb{R}^{d}$, we define

$$
\operatorname{Supp}(f):=\left\{x \in \mathbb{R}^{d}: f(x) \neq 0\right\}
$$

Received by the editors September 8, 2005 and, in revised form, February 2, 2006.

2000 Mathematics Subject Classification. Primary 42C40; Secondary 42C15.

Key words and phrases. Affine systems, wavelets, dilation generator.

The first author was supported by an NSERC grant.

The second author was supported by the Natural Science Foundation of Beijing and the Foundation of Educational Ministry of China. 
The spectrum of $f \in L^{2}\left(\mathbb{R}^{d}\right)$ is the set $\operatorname{Supp}(\hat{f})$ which is defined up to a set of zero measure. We also associate with $f$ the collection $\left\{f_{j, k}\right\}$ defined by

$$
f_{j, k}(\cdot):=|\operatorname{det} D|^{\frac{j}{2}} f\left(D^{j} \cdot-k\right), \quad j \in \mathbb{Z}, k \in \mathbb{Z}^{d},
$$

which we call the affine system with dilation $D$ associated with $f$. More generally, the affine system with dilation $D$ associated with a finite set of functions $\left\{f^{1}, \ldots, f^{m}\right\}$ is the collection $\left\{f_{j, k}^{l}: 1 \leq l \leq m, j \in \mathbb{Z}, k \in \mathbb{Z}^{d}\right\}$. Given a measurable set $S$ in $\mathbb{R}^{d}$, a collection $\left\{S_{\alpha}: \alpha \in \Gamma\right\}$ of measurable subsets of $\mathbb{R}^{d}$ is called a partition of $S$ if

$$
\bigcup_{\alpha \in \Gamma} S_{\alpha}=S \text { and } S_{\alpha_{1}} \cap S_{\alpha_{2}}=\emptyset \text { whenever } \alpha_{1}, \alpha_{2} \in \Gamma, \alpha_{1} \neq \alpha_{2} .
$$

Two measurable sets $S_{1}$ and $S_{2}$ in $\mathbb{R}^{d}$ are called $\mathbb{Z}^{d}$-translation congruent if there exist measurable partitions $\left\{S_{1, k}\right\}_{k \in \mathbb{Z}^{d}}$ of $S_{1}$ and $\left\{S_{2, k}\right\}_{k \in \mathbb{Z}^{d}}$ of $S_{2}$ such that $S_{2, k}=S_{1, k}+k$ for all $k \in \mathbb{Z}^{d}$. Similarly, $S_{1}$ and $S_{2}$ are called $D^{t}$-congruent if there exist measurable partitions $\left\{S_{1, j}^{\prime}\right\}_{j \in \mathbb{Z}}$ of $S_{1}$ and $\left\{S_{2, j}^{\prime}\right\}_{j \in \mathbb{Z}}$ of $S_{2}$ such that $S_{2, j}^{\prime}=\left(D^{t}\right)^{j} S_{1, j}^{\prime}$ for all $j \in \mathbb{Z}$. It is easy to check that $S_{1}$ and $S_{2}$ are $\mathbb{Z}^{d}$-translation congruent if and only if

$$
\sum_{k \in \mathbb{Z}^{d}} \chi_{S_{1}}(\cdot-k)=\sum_{k \in \mathbb{Z}^{d}} \chi_{S_{2}}(\cdot-k),
$$

while $S_{1}$ and $S_{2}$ are $D^{t}$-congruent if and only if

$$
\sum_{j \in \mathbb{Z}} \chi_{S_{1}}\left(\left(D^{t}\right)^{j} \cdot\right)=\sum_{j \in \mathbb{Z}} \chi_{S_{2}}\left(\left(D^{t}\right)^{j} \cdot\right)
$$

where $\chi_{F}$ denotes the characteristic function of the set $F$. A measurable set $E$ in $\mathbb{R}^{d}$ is called a $\mathbb{Z}^{d}$-translation generator if the collection $\left\{E+k: k \in \mathbb{Z}^{d}\right\}$ is a partition of $\mathbb{R}^{d}$. This is clearly equivalent to

$$
\sum_{k \in \mathbb{Z}^{d}} \chi_{E}(\cdot-k)=1
$$

Similarly, $E$ is called a $D^{t}$-dilation generator if $\left\{\left(D^{t}\right)^{j} E: j \in \mathbb{Z}\right\}$ is a partition of $\mathbb{R}^{d}$ or, equivalently, if

$$
\sum_{j \in \mathbb{Z}} \chi_{E}\left(\left(D^{t}\right)^{j} \cdot\right)=1
$$

It is clear that any two $\mathbb{Z}^{d}$-translation generators are $\mathbb{Z}^{d}$-translation congruent and that any two $D^{t}$-dilation generators are $D^{t}$-congruent.

A function $\psi \in L^{2}\left(\mathbb{R}^{d}\right)$ is called a $D$-wavelet if the associated affine system $\left\{\psi_{j, k}: j \in \mathbb{Z}, k \in \mathbb{Z}^{d}\right\}$ is an orthonormal basis for $L^{2}\left(\mathbb{R}^{d}\right)$. Similarly, a finite subset $\left\{\psi^{l}: 1 \leq l \leq m\right\}$ of $L^{2}\left(\mathbb{R}^{d}\right)$ is called a $D$-wavelet collection if the affine system $\left\{\psi_{j, k}^{l}: 1 \leq l \leq m, j \in \mathbb{Z}, k \in \mathbb{Z}^{d}\right\}$ forms an orthonormal basis for $L^{2}\left(\mathbb{R}^{d}\right)$. It was proved in 1, 3, 16 that if $\left\{\psi^{l}: 1 \leq l \leq m\right\}$ of $L^{2}\left(\mathbb{R}^{d}\right)$ is a $2 I_{d^{-}}$wavelet collection associated with an MRA, then $m=2^{d}-1$. Further, it was also proved

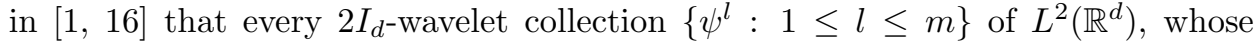
members satisfy a weak smoothness and decay condition on the Fourier transform side, must come from an MRA. Because of these results, the existence of single dyadic wavelets in $L^{2}\left(\mathbb{R}^{d}\right)$ with $d>1$ was unknown until the mid-1990s. Their existence for an arbitrary dilation matrix $D$ was finally proved by Dai, Larson and Speegle in [12] (see also [13]) via the construction of associated wavelet sets. Several 
constructions of such wavelet sets can be found in [4, 5, 6, 14, 15, 17, 18, 19, 21,. For a $d \times d$ dilation matrix $D$, a set $E$ in $\mathbb{R}^{d}$ is called a $D$-wavelet set for $L^{2}\left(\mathbb{R}^{d}\right)$ if $\psi$ defined by $\hat{\psi}=\frac{\chi_{E}}{\sqrt{|E|}}$ is a $D$-wavelet. It is well known that $E$ is a $D$-wavelet set if and only if $E$ is both a $\mathbb{Z}^{d}$-translation and a $D^{t}$-dilation generator of $\mathbb{R}^{d}$. This implies, in particular, that $|E|=1$ if $E$ is a $D$-wavelet set. Additional results on $D$-wavelet sets can be found in 9, 10, 12.

An at most countable subset $\left\{\psi^{l}: l \in \Gamma\right\}$ of $L^{2}\left(\mathbb{R}^{d}\right)$ is said to generate a normalized tight $D$-wavelet frame for $L^{2}\left(\mathbb{R}^{d}\right)$ if

$$
\|f\|^{2}=\sum_{l \in \Gamma} \sum_{j \in \mathbb{Z}} \sum_{k \in \mathbb{Z}^{d}}\left|\left\langle f, \psi_{j, k}^{l}\right\rangle\right|^{2}, \quad f \in L^{2}\left(\mathbb{R}^{d}\right) .
$$

The following two results ([21, Theorem 5 and 6]) due to P. Soardi and D. Weiland show that, on one hand, the support of the Fourier transform of a wavelet cannot have a large hole near the origin, while, on the other hand, if the support of its Fourier transform is not too large, it necessarily must have a hole which is an interval of length one centered at the origin. Related results concerning holes for MRA and non-MRA wavelets can also be found in [2, 6, 8,

Proposition 1.1. Let $\psi$ be a 2-wavelet for $L^{2}(\mathbb{R})$. Then $|\operatorname{Supp}(\hat{\psi}) \cap[-c, c]|>0$ for $c>\frac{1}{2}$.

Proposition 1.2. Let $\psi$ be a 2-wavelet for $L^{2}(\mathbb{R})$ with $|\hat{\psi}|=\chi_{K}$ for some $K \subset$ $[-1,1]$. Then $\left|K \cap\left[-\frac{1}{2}, \frac{1}{2}\right]\right|=0$.

Following the same general philosophy, one of us $(\mathrm{Li})$ proved analogous twodimensional results ([20, Theorem 1.1 and 1.2]). Before stating them, we will define the following sets. If $a, b>0$ are parameters, we let

$$
E^{+}(a, b)=\left\{\left(\xi_{1}, \xi_{2}\right)^{t}:\left|\xi_{1}\right| \leq a,\left|\xi_{1}+\xi_{2}\right| \leq b\right\}
$$

and

$$
E^{-}(a, b)=\left\{\left(\xi_{1}, \xi_{2}\right)^{t}:\left|\xi_{2}\right| \leq a,\left|\xi_{1}-\xi_{2}\right| \leq b\right\} .
$$

Proposition 1.3. Let $M=\left(\begin{array}{rr}1 & 1 \\ 1 & -1\end{array}\right)$, and let $\psi$ be an $M$-wavelet. Then, for $c>\frac{1}{2}$, we have $\left|\operatorname{Supp}(\hat{\psi}) \cap E^{+}(c, c)\right|>0$ and $\left|\operatorname{Supp}(\hat{\psi}) \cap E^{-}(c, c)\right|>0$.

Proposition 1.4. Let $M=\left(\begin{array}{rr}1 & 1 \\ 1 & -1\end{array}\right)$ and let $\psi$ be an $M$-wavelet satisfying that $|\hat{\psi}|=\chi_{K}$ for some measurable set $K$.

(a) If $K \subset E^{+}\left(\frac{1}{2}, 1\right)$, then $\left|K \cap E^{+}\left(\frac{1}{2}, \frac{1}{2}\right)\right|=0$.

(b) If $K \subset E^{-}\left(\frac{1}{2}, 1\right)$, then $\left|K \cap E^{-}\left(\frac{1}{2}, \frac{1}{2}\right)\right|=0$.

Note the following: the set

$$
E_{1}:=\left[-1,-\frac{1}{2}\right] \cup\left[\frac{1}{2}, 1\right]
$$

is a 2 -wavelet set for $L^{2}(\mathbb{R})$ (the so-called Shannon wavelet-set), while the intervals $[-1,1]$ and $\left[-\frac{1}{2}, \frac{1}{2}\right]$ appearing in Proposition 1.2 can be expressed as

$$
[-1,1]=\bigcup_{j \leq 0} 2^{j} E_{1}, \quad\left[-\frac{1}{2}, \frac{1}{2}\right]=\bigcup_{j<0} 2^{j} E_{1} .
$$


At the same time, the interval $[-c, c]$ with $c>\frac{1}{2}$ appearing in Proposition 1.1 can be written as

$$
[-c, c]=\lambda \bigcup_{j<0} 2^{j} E_{1}
$$

with $\lambda>1$. Similarly, both

$$
E^{+}=\left\{\left(\xi_{1}, \xi_{2}\right)^{t}:\left|\xi_{1}\right| \leq \frac{1}{2}, \frac{1}{2} \leq\left|\xi_{1}+\xi_{2}\right| \leq 1\right\}
$$

and

$$
E^{-}=\left\{\left(\xi_{1}, \xi_{2}\right)^{t}:\left|\xi_{2}\right| \leq \frac{1}{2}, \frac{1}{2} \leq\left|\xi_{1}-\xi_{2}\right| \leq 1\right\}
$$

are $M$-wavelet sets in $L^{2}\left(\mathbb{R}^{2}\right)$ for the matrix $M=\left(\begin{array}{ll}1 & 1 \\ 1 & -1\end{array}\right)$ appearing in Proposition 1.3 and Proposition 1.4. Furthermore, in relation with Proposition 1.4, we have

$$
E^{+}\left(\frac{1}{2}, 1\right)=\bigcup_{j \leq 0} M^{j} E^{+} \text {and } E^{-}\left(\frac{1}{2}, 1\right)=\bigcup_{j \leq 0} M^{j} E^{-},
$$

while

$$
E^{+}\left(\frac{1}{2}, \frac{1}{2}\right)=\bigcup_{j<0} M^{j} E^{+} \quad \text { and } \quad E^{-}\left(\frac{1}{2}, \frac{1}{2}\right)=\bigcup_{j<0} M^{j} E^{-} .
$$

Similarly, for the sets appearing in Proposition 1.3, whenever $c>\frac{1}{2}$,

$$
E^{+}(c, c)=\lambda \bigcup_{j<0} M^{-j} E^{+} \quad \text { and } \quad E^{-}(c, c)=\lambda \bigcup_{j<0} M^{-j} E^{-}
$$

for $\lambda=2 c>1$. Inspired by these observations, it is natural for us to ask whether or not the previous results can be extended to the case of a wavelet associated with a general expansive matrix in $\mathbb{R}^{d}$ and of any wavelet set corresponding to the same dilation matrix. Our main goal will be to give a positive answer to that question. In fact our results make even weaker assumptions since, instead of wavelet sets, they are stated for sets that are $D^{t}$-dilation generators of measure larger (resp. smaller) than or equal to one. The main results of this paper are stated below. We formulate the two next theorems for finitely generated wavelet systems as opposed to singly generated ones. Note that, in the first one of these, the dilation matrix is assumed to be integral, i.e. its entries must be integers.

Theorem 1.5. Let $D$ be a $d \times d$ integral dilation matrix, let $E$ be a $D^{t}$-dilation generator with $|E| \geq 1$, and let $\left\{\psi^{l}: 1 \leq l \leq m\right\}$ be a $D$-wavelet collection. Then, to every $\tau$ with $\tau>m^{\frac{1}{d}}$, there corresponds an $l$ with $1 \leq l \leq m$ such that

$$
\left|\operatorname{Supp}\left(\hat{\psi}^{l}\right) \cap\left(\tau \bigcup_{j<0}\left(D^{t}\right)^{j} E\right)\right|>0 .
$$

Obviously, the previous remarks show that Proposition 1.1 and Proposition 1.3 are particular cases of Theorem 1.5. We can also use it to obtain the following one-dimensional result which generalizes Proposition 1.1.

Corollary 1.6. If $\psi$ is a 2-wavelet for $L^{2}(\mathbb{R})$, then $|\operatorname{Supp}(\hat{\psi}) \cap[\alpha, \beta]|>0$ for $-1<\alpha<0$, and $\beta>1+\alpha$. 
Indeed, if we take $\gamma=\frac{\beta}{\beta-\alpha}$ and $E=[2 \gamma-2, \gamma-1] \cup[\gamma, 2 \gamma]$, then $E$ is a 2 -wavelet set for $L^{2}(\mathbb{R})$ since it is both a $\mathbb{Z}$-translation and a 2-dilation generator of $\mathbb{R}$. Note that $[\alpha, \beta]=\tau \bigcup_{j<0} 2^{j} E$ with $\tau=\beta-\alpha>1$. It follows from Theorem 1.5 that $|\operatorname{Supp}(\hat{\psi}) \cap[\alpha, \beta]|>0$.

The following result concerns orthonormal affine systems having more than one generator and which might not generate the whole space $L^{2}\left(\mathbb{R}^{d}\right)$.

Theorem 1.7. Let $D$ be a $d \times d$ dilation matrix, let $E$ be a $D^{t}$-dilation generator of measure not greater than 1 , and let $\left\{\psi_{j, k}^{l}: 1 \leq l \leq m, j \in \mathbb{Z}, k \in \mathbb{Z}^{d}\right\}$ be an orthonormal affine system with dilation $D$ in $L^{2}\left(\mathbb{R}^{d}\right)$. Suppose, furthermore, that $m>1$. Then,

$$
\left|\operatorname{Supp}\left(\hat{\psi}^{l}\right) \cap\left(\bigcup_{j>0}\left(D^{t}\right)^{j} E\right)\right|>0,
$$

for some $l$ with $1 \leq l \leq m$.

Theorem 1.7 shows that, for any $D^{t}$-dilation generator of measure not greater than 1 , in particular, for any $D$-wavelet set $E$, and any $D$-wavelet collection $\left\{\psi^{l}\right.$ : $1 \leq l \leq m\}$ with $m>1$, it is impossible that $\operatorname{Supp}\left(\hat{\psi}^{l}\right)$ be a subset of $\bigcup_{j \leq 0}\left(D^{t}\right)^{j} E$ for every $1 \leq l \leq m$. Interestingly, this last result can also be obtained from a result of M. Bownik ([7, Theorem 3.7]) on generalized multiresolution analyses (GMRA) applied to the situation where $\hat{V}_{1}=L^{2}\left(\bigcup_{j \leq 0}\left(D^{t}\right)^{j} E\right.$ ). (See more particularly the remark following Corollary 3.8 in [7.) The following theorem shows that Theorem 1.7 is also true when $m=1$, except in the case where $E$ is actually a $D$-wavelet set.

Theorem 1.8. Let $D$ be a $d \times d$ dilation matrix, let $E$ be a $D^{t}$-dilation generator of measure not greater than 1 , and let $\left\{\psi_{j, k}: j \in \mathbb{Z}, k \in \mathbb{Z}^{d}\right\}$ be an orthonormal affine system with dilation $D$ in $L^{2}\left(\mathbb{R}^{d}\right)$. Suppose, furthermore, that the inclusion $\operatorname{Supp}(\hat{\psi}) \subset \bigcup_{j \leq 0}\left(D^{t}\right)^{j} E$ holds. Then, $E$ is a D-wavelet set and $|\hat{\psi}|=\chi_{E}$.

In relation with this last result, we point out that if a function $\psi \in L^{2}\left(\mathbb{R}^{d}\right)$ satisfies $|\hat{\psi}|=\chi_{E}$, for some $D$-wavelet set $E$, then $\psi$ is a $D$-wavelet for $L^{2}\left(\mathbb{R}^{d}\right)$.

\section{Proofs of the main Results}

We will need the following lemma.

Lemma 2.1. Let $\tau>0$, let $D$ be a $d \times d$ dilation matrix, and let $E$ be a $D^{t}$-dilation generator. Define $\phi^{(s)}, s \in \mathbb{Z}^{d}$, by

$$
\hat{\phi}^{(s)}=\chi_{\tau E \cap\left([0,1]^{d}+s\right)} .
$$

Then, the collection $\left\{\phi^{(s)}: s \in \mathbb{Z}^{d}\right\}$ generates a normalized tight $D$-wavelet frame for $L^{2}\left(\mathbb{R}^{d}\right)$.

Proof. Observing that

$$
\hat{\phi}_{j, k}^{(s)}(\xi)=|\operatorname{det} D|^{-\frac{j}{2}} \hat{\phi}^{(s)}\left(\left(D^{t}\right)^{-j} \xi\right) e^{-2 \pi i\left\langle k,\left(D^{t}\right)^{-j} \xi\right\rangle}, \quad \xi \in \mathbb{R}^{d},
$$


we have, for $f \in L^{2}\left(\mathbb{R}^{d}\right)$

$$
\begin{aligned}
& \sum_{s \in \mathbb{Z}^{d}} \sum_{j \in \mathbb{Z}} \sum_{k \in \mathbb{Z}^{d}}\left|\left\langle f, \phi_{j, k}^{(s)}\right\rangle\right|^{2} \\
& =\sum_{s \in \mathbb{Z}^{d}} \sum_{j \in \mathbb{Z}} \sum_{k \in \mathbb{Z}^{d}}|\operatorname{det} D|^{-j}\left|\int_{\mathbb{R}^{d}} \hat{f}(\xi) \overline{\hat{\phi}^{(s)}\left(\left(D^{t}\right)^{-j} \xi\right)} e^{2 \pi i\left\langle k,\left(D^{t}\right)^{-j} \xi\right\rangle} d \xi\right|^{2} \\
& =\sum_{s \in \mathbb{Z}^{d}} \sum_{j \in \mathbb{Z}} \sum_{k \in \mathbb{Z}^{d}}|\operatorname{det} D|^{j}\left|\int_{\mathbb{R}^{d}} \hat{f}\left(\left(D^{t}\right)^{j} \xi\right) \overline{\hat{\phi}^{(s)}(\xi)} e^{2 \pi i\langle k, \xi\rangle} d \xi\right|^{2} \\
& =\sum_{s \in \mathbb{Z}^{d}} \sum_{j \in \mathbb{Z}} \sum_{k \in \mathbb{Z}^{d}}|\operatorname{det} D|^{j}\left|\int_{\tau E \cap\left([0,1]^{d}+s\right)} \hat{f}\left(\left(D^{t}\right)^{j} \xi\right) e^{2 \pi i\langle k, \xi\rangle} d \xi\right|^{2} .
\end{aligned}
$$

Applying Parseval's theorem on the interval $[0,1]^{d}+s$, we can rewrite this last expression as

$$
\begin{aligned}
& \sum_{s \in \mathbb{Z}^{d}} \sum_{j \in \mathbb{Z}}|\operatorname{det} D|^{j} \int_{\tau E \cap\left([0,1]^{d}+s\right)}\left|\hat{f}\left(\left(D^{t}\right)^{j} \xi\right)\right|^{2} d \xi \\
& =\sum_{j \in \mathbb{Z}}|\operatorname{det} D|^{j} \int_{\tau E}\left|\hat{f}\left(\left(D^{t}\right)^{j} \xi\right)\right|^{2} d \xi \\
& =\sum_{j \in \mathbb{Z}} \int_{\tau\left(D^{t}\right)^{j} E}|\hat{f}(\xi)|^{2} d \xi=\int_{\mathbb{R}^{d}}|\hat{f}(\xi)|^{2} d \xi=\|f\|^{2},
\end{aligned}
$$

which proves the lemma.

Proof of Theorem 1.5. Suppose that, for some $\tau>0$,

$$
\left|\operatorname{Supp}\left(\hat{\psi}^{l}\right) \cap\left(\tau \bigcup_{j<0}\left(D^{t}\right)^{j} E\right)\right|=0, \quad 1 \leq l \leq m .
$$

It suffices to show that $\tau \leq m^{\frac{1}{d}}$ under these conditions. Let $\phi^{(s)}$ be defined as in Lemma 2.1. Then

$$
\left\|\phi^{(s)}\right\|^{2}=\left\|\hat{\phi}^{(s)}\right\|^{2}=\left|\tau E \cap\left([0,1]^{d}+s\right)\right|
$$

and

$$
\sum_{s \in \mathbb{Z}^{d}}\left\|\phi^{(s)}\right\|^{2}=|\tau E|=\tau^{d}|E|
$$


Since $\left\{\psi^{l}: 1 \leq l \leq m\right\}$ is a $D$-wavelet collection, we have

$$
\begin{aligned}
& \tau^{d}|E|=\sum_{s \in \mathbb{Z}^{d}}\left\|\phi^{(s)}\right\|^{2}=\sum_{s \in \mathbb{Z}^{d}} \sum_{l=1}^{m} \sum_{j \in \mathbb{Z}} \sum_{k \in \mathbb{Z}^{d}}\left|\left\langle\phi^{(s)}, \psi_{j, k}^{l}\right\rangle\right|^{2} \\
& =\left.\left.\sum_{s \in \mathbb{Z}^{d}} \sum_{l=1}^{m} \sum_{j \in \mathbb{Z}} \sum_{k \in \mathbb{Z}^{d}}\left|\int_{\mathbb{R}^{d}} \hat{\phi}^{(s)}(\xi)\right| \operatorname{det} D\right|^{-\frac{j}{2}} \overline{\hat{\psi}^{l}\left(\left(D^{t}\right)^{-j} \xi\right)} e^{2 \pi i\left\langle k,\left(D^{t}\right)^{-j} \xi\right\rangle} d \xi\right|^{2} \\
& =\sum_{s \in \mathbb{Z}^{d}} \sum_{l=1}^{m} \sum_{j \in \mathbb{Z}} \sum_{k \in \mathbb{Z}^{d}}|\operatorname{det} D|^{j}\left|\int_{\mathbb{R}^{d}} \hat{\phi}^{(s)}\left(\left(D^{t}\right)^{j} \xi\right) \overline{\hat{\psi}^{l}(\xi)} e^{2 \pi i\langle k, \xi\rangle} d \xi\right|^{2} \\
& =\sum_{s \in \mathbb{Z}^{d}} \sum_{l=1}^{m} \sum_{j \leq 0} \sum_{k \in \mathbb{Z}^{d}}|\operatorname{det} D|^{j}\left|\int_{\mathbb{R}^{d}} \hat{\phi}^{(s)}\left(\left(D^{t}\right)^{j} \xi\right) \overline{\hat{\psi}^{l}(\xi)} e^{2 \pi i\langle k, \xi\rangle} d \xi\right|^{2},
\end{aligned}
$$

where in the last equality we used (2.1) and the fact that

$$
\operatorname{Supp}\left(\hat{\phi}^{(s)}\left(\left(D^{t}\right)^{j} \cdot\right)\right) \subset\left(D^{t}\right)^{-j} \tau E, \quad j \in \mathbb{Z} .
$$

Also, taking into account the fact that $D$ is an integral matrix and that

$$
\mathbb{Z}^{d}=\left\{k, k \in \mathbb{Z}^{d}\right\} \subset\left\{D^{j} k, k \in \mathbb{Z}^{d}\right\}, \quad \text { for any } j \leq 0,
$$

leads to

$$
\begin{aligned}
\tau^{d}|E| & \leq \sum_{s \in \mathbb{Z}^{d}} \sum_{l=1}^{m} \sum_{j \leq 0} \sum_{k \in \mathbb{Z}^{d}}|\operatorname{det} D|^{j}\left|\int_{\mathbb{R}^{d}} \hat{\phi}^{(s)}\left(\left(D^{t}\right)^{j} \xi\right) \overline{\hat{\psi}^{l}(\xi)} e^{\left.2 \pi i\left\langle D^{j} k, \xi\right)\right\rangle} d \xi\right|^{2} \\
& \leq \sum_{s \in \mathbb{Z}^{d}} \sum_{l=1}^{m} \sum_{j \in \mathbb{Z}} \sum_{k \in \mathbb{Z}^{d}}|\operatorname{det} D|^{j}\left|\int_{\mathbb{R}^{d}} \hat{\phi}^{(s)}\left(\left(D^{t}\right)^{j} \xi\right) \overline{\hat{\psi}^{l}(\xi)} e^{\left.2 \pi i\left\langle k,\left(D^{t}\right)^{j} \xi\right)\right\rangle} d \xi\right|^{2} \\
& =\sum_{l=1}^{m} \sum_{s \in \mathbb{Z}^{d}} \sum_{j \in \mathbb{Z}} \sum_{k \in \mathbb{Z}^{d}}\left|\left\langle\phi_{j, k}^{(s)}, \psi^{l}\right\rangle\right|^{2} .
\end{aligned}
$$

It follows that $\tau^{d} \leq \sum_{l=1}^{m}\left\|\psi^{l}\right\|^{2}=m$ by Lemma 2.1. The proof is completed.

Proof of Theorem 1.7. We will argue by contradiction. Suppose that

$$
\operatorname{Supp}\left(\hat{\psi}^{l}\right) \subset\left(\bigcup_{j \leq 0}\left(D^{t}\right)^{j} E\right), \quad 1 \leq l \leq m .
$$

Let $\|.\|_{d}$ denote the usual matrix norm of a $d \times d$ matrix. Due to the fact that $\lim _{j \rightarrow-\infty}\left\|\left(D^{t}\right)^{j}\right\|_{d}^{-\frac{1}{j}}<1$, there exists a constant $c_{0} \geq 1$ such that $\left\|\left(D^{t}\right)^{j}\right\|_{d} \leq c_{0}$ for $j$ with $j \leq 0$. Let $c:=2 c_{0}$ and define the sets $S_{p}$ by

$$
S_{p}=\left[-\frac{1}{2 c}, \frac{1}{2 c}\right]^{d}+\frac{p}{c}, \quad p \in \mathbb{Z}^{d}
$$

Then, $\left\{S_{p}: p \in \mathbb{Z}^{d}\right\}$ is a partition of $\mathbb{R}^{d}$, and $\left(D^{t}\right)^{j} S_{p}$ is $\mathbb{Z}^{d}$-translation congruent to a subset of $\left[-\frac{1}{2}, \frac{1}{2}\right]^{d}$ for every $j$ with $j \leq 0$. Define $\psi_{E}$ by $\hat{\psi}_{E}=\chi_{E}$. Then observing that the collection $\left\{\hat{\psi}_{j, k}^{l}: 1 \leq l \leq m, j \in \mathbb{Z}, k \in \mathbb{Z}^{d}\right\}$ is also an 
orthonormal system in $L^{2}\left(\mathbb{R}^{d}\right)$ leads to

$$
\begin{aligned}
& 1 \geq\left\|\hat{\psi}_{E}\right\|^{2}=\sum_{p \in \mathbb{Z}^{d}} \int_{\mathbb{R}^{d}}\left|\hat{\psi}_{E}(\xi) \chi_{S_{p}}(\xi)\right|^{2} d \xi \\
& \geq \sum_{p \in \mathbb{Z}^{d}} \sum_{l=1}^{m} \sum_{j \in \mathbb{Z}} \sum_{k \in \mathbb{Z}^{d}}\left|\left\langle\hat{\psi}_{E} \chi_{S_{p}}, \hat{\psi}_{j, k}^{l}\right\rangle\right|^{2} \\
& =\left.\left.\sum_{p \in \mathbb{Z}^{d}} \sum_{l=1}^{m} \sum_{j \in \mathbb{Z}} \sum_{k \in \mathbb{Z}^{d}}\left|\int_{E \cap S_{p}}\right| \operatorname{det} D\right|^{-\frac{j}{2}} \overline{\hat{\psi}^{l}\left(\left(D^{t}\right)^{-j} \xi\right)} e^{2 \pi i\left\langle k,\left(D^{t}\right)^{-j} \xi\right\rangle} d \xi\right|^{2} \\
& =\sum_{p \in \mathbb{Z}^{d}} \sum_{l=1}^{m} \sum_{j \in \mathbb{Z}}|\operatorname{det} D|^{j} \sum_{k \in \mathbb{Z}^{d}}\left|\int_{\left(D^{t}\right)^{-j}\left(E \cap S_{p}\right)} \overline{\hat{\psi}^{l}(\xi)} e^{2 \pi i\langle k, \xi\rangle} d \xi\right|^{2} \\
& =\sum_{p \in \mathbb{Z}^{d}} \sum_{l=1}^{m} \sum_{j \geq 0}|\operatorname{det} D|^{j} \sum_{k \in \mathbb{Z}^{d}}\left|\int_{\left(D^{t}\right)^{-j}\left(E \cap S_{p}\right)} \overline{\hat{\psi}^{l}(\xi)} e^{2 \pi i\langle k, \xi\rangle} d \xi\right|^{2},
\end{aligned}
$$

where (2.2) was used in the last equality. Applying Plancherel's formula gives

$$
\begin{aligned}
1 & \geq \sum_{p \in \mathbb{Z}^{d}} \sum_{l=1}^{m} \sum_{j \geq 0}|\operatorname{det} D|^{j} \int_{\left(D^{t}\right)^{-j}\left(E \cap S_{p}\right)}\left|\hat{\psi}^{l}(\xi)\right|^{2} d \xi \\
& =\sum_{j \geq 0}|\operatorname{det} D|^{j} \sum_{l=1}^{m} \int_{\left(D^{t}\right)^{-j} E}\left|\hat{\psi}^{l}(\xi)\right|^{2} d \xi .
\end{aligned}
$$

Because of (2.2) and the fact that $E$ is a $D^{t}$-dilation generator, we have

$$
1=\frac{1}{m} \sum_{l=1}^{m}\left\|\hat{\psi}^{l}\right\|^{2}=\sum_{j \geq 0} \frac{1}{m} \sum_{l=1}^{m} \int_{\left(D^{t}\right)^{-j} E}\left|\hat{\psi}^{l}(\xi)\right|^{2} d \xi .
$$

This together with (2.3) leads to

$$
\sum_{j \geq 0}\left(|\operatorname{det} D|^{j}-\frac{1}{m}\right) \sum_{l=1}^{m} \int_{\left(D^{t}\right)^{-j} E}\left|\hat{\psi}^{l}(\xi)\right|^{2} d \xi \leq 0 .
$$

Since $|\operatorname{det} D|>1$ and $m \geq 2$, it follows immediately that

$$
\left|\operatorname{Supp}\left(\hat{\psi}^{l}\right) \cap\left(\bigcup_{j \leq 0}\left(D^{t}\right)^{j} E\right)\right|=0
$$

for $l$ with $1 \leq l \leq m$. Again using (2.2), we conclude that $\hat{\psi}^{l}=0$ a.e. on $\mathbb{R}^{d}$ for $l$ with $1 \leq l \leq m$, which yields a contradiction. The proof is completed.

Proof of Theorem 1.8, Proceeding as in the proof of Theorem 1.7, we see that, when $m=1$, (2.4) becomes

$$
\sum_{j \geq 0}\left(|\operatorname{det} D|^{j}-1\right) \int_{\left(D^{t}\right)^{-j} E}|\hat{\psi}(\xi)|^{2} d \xi \leq 0 .
$$

Since $|\operatorname{det} D|>1$, we obtain immediately that

$$
\int_{\left(D^{t}\right)^{j} E}|\hat{\psi}(\xi)|^{2} d \xi=0
$$


for all $j \in \mathbb{Z}$ with $j<0$, which implies, using our hypothesis on the spectrum of $\psi$, that $\operatorname{Supp}(\hat{\psi}) \subset E$. Since the sequence $\{\psi(\cdot-n)\}_{n \in \mathbb{Z}}$ is orthonormal, it follows that

$$
\sum_{k \in \mathbb{Z}^{d}}|\hat{\psi}(\cdot+k)|^{2}=1
$$

This implies that $|\hat{\psi}| \leq \chi_{E}$ almost everywhere. Integrating both sides of this last inequality, we obtain

$$
1=\int_{\mathbb{R}^{d}}|\hat{\psi}(\xi)|^{2} d \xi \leq \int_{\mathbb{R}^{d}} \chi_{E}(\xi) d \xi=|E| .
$$

Since $|E| \leq 1$ by hypothesis, it follows that $|\hat{\psi}|=\chi_{E}$. This fact, together with $(2.5)$, shows that the $\mathbb{Z}^{d}$-translates of $E$ tiles $\mathbb{R}^{d}$ and $E$ is thus a $D$-wavelet set. This completes the proof.

\section{ACKNOWLEDGEMENT}

The authors would like to thank the referee for carefully reviewing this manuscript and, in particular, for pointing out relevant additional references.

\section{REFERENCES}

[1] P. Auscher, Solution of two problems on wavelets, J. Geom. Anal., 5 (1995), 181-236. MR.1341029 (96g:42016)

[2] L. W. Baggett, An abstract interpretation of the wavelet dimension function using group representations, J. Funct. Anal., 173 (2000), 1-20. MR.1760275 (2001j:42028)

[3] L. Baggett, A. Carey, W. Moran and P. Ohring, General existence theorems for orthonormal wavelets, an abstract approach, Publ. Res. Inst. Math. Sci. Kyoto Univ., 31 (1995), 95-111. MR.1317525 (96c:42060)

[4] L. W. Baggett, H. A. Medina, K. D. Merrill, Generalized multi-resolution analyses and a construction procedure for all wavelet sets in $\mathbb{R}^{n}$, J. Fourier Anal. Appl., 5 (1999), 563-573. MR.1752590 (2001f:42055)

[5] J. J. Benedetto and M. T. Leon, The construction of single wavelets in $d$-dimensions, $J$. Geom. Anal., 11 (2001), 1-15. MR1829345(2002g:42045)

[6] J. J. Benedetto and S. Sumetkijakan, A fractal set constructed from a class of wavelet sets, Contemp. Math., 19-35, 313, Amer. Math. Soc., Providence, RI, 2002. MR1940987 (2003i:42048)

[7] M. Bownik, Riesz wavelets and generalized multiresolution analyses, Appl. Comput. Harmon. Anal., 14 (2003), 181-194. MR1984546 (2004d:42057)

[8] L. Brandolini, G. Garrigós, Z. Rzeszotnik, G. Weiss, The behaviour at the origin of a class of band-limited wavelets, in The functional and harmonic analysis of wavelets and frames (San Antonio, TX) (L. Baggett and D. Larson, eds.), Contemp. Math. 247, Amer. Math. Soc., Providence, RI (1999), 75-91. MR.1738086 (2001b:42045)

[9] X. Dai, Y. Diao, Q. Gu and D. Han, Wavelets with frame multiresolution analysis, J. Fourier Anal. Appl., 9 (2003), 39-48. MR.1953071 (2003j:42033)

[10] X. Dai, Y. Diao, Q. Gu and D. Han, The existence of subspace wavelet sets, J. Comput. Appl. Math., 155 (2003), 83-90. MR 1992291 (2005c:42034)

[11] X. Dai and D. Larson, Wandering vectors for unitary systems and orthogonal wavelets, Mem. Amer. Math. Soc. 134 (1998), no. 640. MR1432142 (98m:47067)

[12] X. Dai, D. Larson and D. M. Speegle, Wavelet sets in $\mathbb{R}^{n}$, J. Fourier Anal. Appl., 3 (1997), 451-456. MR:1468374 (98m:42048)

[13] X. Dai, D. Larson and D. M. Speegle, Wavelet sets in $\mathbb{R}^{n}$ II, Contemp. Math., 216 (1998), 15-40. MR $1614712(99 \mathrm{~d}: 42054)$

[14] X. Fang and X. Wang, Construction of minimally supported frequency wavelets, J. Fourier Anal. Appl., 2 (1996), 315-327. MR.1395767(97d:42030) 
[15] J.-P. Gabardo and X. Yu, Construction of wavelet sets with certain self-similarity properties, J. Geom. Anal., 14 (2004), 629-651. MR2111421 (2005h:42070)

[16] G. Gripenberg, A necessary and sufficient condition for the existence of a father wavelet, Studia Math., 114 (1995), 207-226. MR1338828 (96d:42049)

[17] E. Hernandez, X. Wang and G. Weiss, Smoothing minimally supported frequency wavelets: Part I, J. Fourier Anal. Appl., 2 (1996), 329-340. MR1395768 (97h:42015)

[18] E. Hernandez, X. Wang and G. Weiss, Smoothing minimally supported frequency wavelets: Part II, J. Fourier Anal. Appl., 3 (1997), 23-41. MR1428814(98b:42049)

[19] E. J. Ionascu, A new construction of wavelet sets, Real Anal. Exchange, 28 (2003), 593-609. MR2010340 (2004g:42040)

[20] Y.-Z. Li, On the holes of a class of bidimensional nonseparable wavelets, J. Approx. Theory, 125 (2003), 151-168. MR2019606 (2004i:42035)

[21] P. M. Soardi and D. Weiland, Single wavelets in $n$-dimensions, J. Fourier Anal. Appl., 4 (1998), 299-315. MR1650988 (99k:42067)

Department of Mathematics and Statistics, McMaster University, Hamilton, OnTARIO, CANADA L8S $4 \mathrm{~K} 1$

E-mail address: gabardo@mcmaster.ca

Department of Applied Mathematics, Beijing University of Technology, Beijing, 100022, People's Republic of China

E-mail address: yzlee@bjut.edu.cn 